\title{
The Protective Effect of a Puerariae flos Extract (Thomsonide) against Ethanol-Induced Gastric Lesions in Rats
}

\author{
Takashi Yamazaki1 $^{*}$, Yoshijiro Nakajima1, Junei Kinjo² ${ }^{2}$ Toshihiro Nohara ${ }^{3}$ \\ ${ }^{1}$ Tsukuba Research Institute, Ohta's Isan Co. Ltd., Ibaraki, Japan \\ ${ }^{2}$ Department of Pharmacognosy, Faculty of Pharmaceutical Sciences, Fukuoka University, Fukuoka, Japan \\ ${ }^{3}$ Department of Natural Medicines, Faculty of Pharmaceutical Sciences, Sojo University, Kumamoto, Japan \\ Email: "t_yamazaki@ohta-isan.co.jp
}

Received 6 March 2016; accepted 28 May 2016; published 31 May 2016

Copyright (C) 2016 by authors and Scientific Research Publishing Inc.

This work is licensed under the Creative Commons Attribution International License (CC BY).

http://creativecommons.org/licenses/by/4.0/

(c) (i) Open Access

\begin{abstract}
Objectives: Puerariae flos has popularly been used to treat alcoholic disorders. However, the effect of Puerariae flos on alcoholic disorders in the gastrointestinal system has not been identified. We investigated the protective effect of an extract of Puerariae flos against the murine gastric mucosa. Methods: Thomsonide, the extracts containing large amounts of isoflavonoid and triterpenoid saponin, was obtained from Puerriae flos via Diaion HP-20 column chromatography using water and $\mathbf{9 9 . 5 \%}$ ethanol. It was investigated whether thomsonide, as well as geranylgeranylacetone (teprenone), a popular anti-ulcer agent developed in Japan, had a cytoprotective effect that might be related to endogenous prostaglandins, which played an important role in preventing gastric mucosal lesions. Results: Thomsonide and teprenone inhibited ethanol-induced gastric lesions. Furthermore, thomsonide increased the production of $\mathrm{PGE}_{2}$ and $6-\mathrm{ketoPGF}_{1} \alpha$, a stable metabolite of $\mathrm{PGI}_{2}$, in the gastric mucosa, and protective effects of thomsonide, as well as teprenone, against ethanol-induced gastric lesions were attenuated by pretreatment with indomethacin. Conclusions: These findings suggest that thomsonide, as well as teprenone, has the gastro protective effect which may be related to the cytoprotective activity of endogenous prostaglandins. The results of this study also suggest that the gastro protective effect of thomsonide may partially mitigate alcoholic disorders in the gastrointestinal tract, and support our pharmacological belief that Puerariae flos is useful for treatment of alcoholic disorders.
\end{abstract}

\section{Keywords}

Alcoholic Disorders, Ethanol, Gastric Mucosal Lesions, Prostaglandins, Puerariae flos

\footnotetext{
"Corresponding author.
}

How to cite this paper: Yamazaki, T., Nakajima, Y., Kinjo, J. and Nohara, T. (2016) The Protective Effect of a Puerariae flos Extract (Thomsonide) against Ethanol-Induced Gastric Lesions in Rats. Pharmacology \& Pharmacy, 7, 208-215. 


\section{Introduction}

Puerariae flos, the dried flower of Pueraria thomsonii Benth. (Leguminosae), is known to counteract the effects of orally consumed ethanol [1]. Kakkakaiseito is one of the well-known Chinese medicines prepared from $\mathrm{Pu}$ erariae flos and several other herbs. Kakkakaiseito has traditionally been used to treat alcoholic disorders. Chemical studies have shown that Puerariae flos contains various isoflavonoids and triterpenoid saponins [2] [3]. Yamazaki et al. have reported that an extract of Puerariae flos promotes elimination of acetaldehyde from human blood [4], and improves learning and memory impairments, which are induced by ethanol or scopolamine [5]. However, despite the fact that ethanol is widely recognized to induce gastric mucosal lesions in mammals, only few extensive pharmacological studies have been conducted to evaluate the effect of Puerariae flos on alcoholic disorders in the gastrointestinal system. A large amount of alcohol consumption induces gastric mucosal lesions, while Puerariae flos has been used in traditional Chinese herbal medicine to treat alcoholic disorders, under the circumstances we investigated the possibility of Puerariae flos affording gastro protection against ethanol-induced gastric mucosal lesions. The present study was designed to investigate whether the extract of Puerariae flos, as well as geranylgeranylacetone, a popular anti-ulcer agent developed in Japan [6], had a cytoprotective effect that might be related to endogenous prostaglandins (PGs), which played an important role in preventing gastric mucosal lesions. In addition, since indomethacin, a cyclo-oxygenase (COX) inhibitor, has been used to determine the role of endogenous PGs, we evaluate the gastro protective effect of the Puerariae flos extract against ethanol-induced gastric mucosal lesions in indomethacin-pretreated rats.

\section{Materials and Methods}

\subsection{Plant Materials}

\subsubsection{Extract Preparations}

Puerariae flos, the air-dried flowers of Pueraria thomsonii, was purchased from Mikuni Co. (Osaka, Japan). A dried voucher specimen (PFT0013) was deposited in our laboratory for future reference. Preparations for extracts were made in the factory in Mikuni Co.; dry flowers of the plant $(1.5 \mathrm{~kg})$ were mixed with $30 \mathrm{~L}$ of boiling water, extracted for $2 \mathrm{~h}$ at $95^{\circ} \mathrm{C}$, and filtered. The filtrate was concentrated and spray drying was carried out to obtain the aqueous extract ( $400 \mathrm{~g}$ ). The aqueous extract was put into $2 \mathrm{~L}$ of boiling water, and was centrifuged. The supernatant obtained was subjected to Diaion HP-20 column chromatography using water and 99.5\% ethanol to give ethanolic elution (6 L) containing large amounts of isoflavonoid and triterpenoid saponin. The ethanolic elution was then filtered, and the filtrate was further concentrated under reduced pressure to obtain $60 \mathrm{~g}$ of dried brownish powder. It had a characteristic odor and bitter taste, and was named as thomsonide (Lot 9,954,111). Thomsonide was used for the following experiments.

\subsubsection{Analysis of Isoflavonoids and Triterpenoid Saponins in Thomsonide}

Simultaneous analyses of isoflavonoids and triterpenoid saponins in thomsonide were performed by using HPLC coupled with an evaporative light scattering detector (ELSD) according to the method of Niiho et al. [7], as follows: $20 \mathrm{mg}$ of thomsonide was dissolved in $6 \mathrm{~mL}$ volume of $80 \%$ ethanol, and then $10 \mu \mathrm{L}$ of the ethanolic elution made by a membrane filter (Millipore Co., USA) was applied to HPLC-ELSD instrument system for analyses of isoflavonoids and triterpenoid saponins. HPLC-ELSD instrument system was equipped with a column heater (U-620, Sugai), a pump unit (CCPM-II, Tosoh), and ELSD (Model 300s, SofTA, USA). Analytical con-

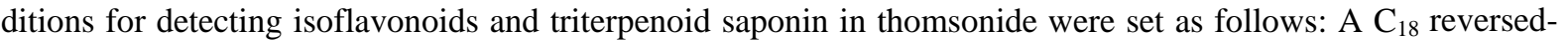
phase column (COSMOSIL 5C18-PAQ $250 \mathrm{~mm} \times$ 3-mm i.d., Nacalai Tesque, Kyoto, Japan); a spray chamber at $40^{\circ} \mathrm{C}$, and a drift tube at $60^{\circ} \mathrm{C}$. The mobile phases were solvent A (trifluoroacetic acid (TFA): water $=0.05: 100$ ) and solvent $B$ (acetonitrile: water:TFA $=150: 100: 0.05$ ). The following solvent gradients were applied: from $100 \% \mathrm{~A}$ and $0 \% \mathrm{~B}$ to $17 \% \mathrm{~A}$ and $83 \% \mathrm{~B}$ within $30 \mathrm{~min}$, and $0 \% \mathrm{~A}$ to $100 \% \mathrm{~B}$ within $45 \mathrm{~min}$. The flow rate was $0.5 \mathrm{~mL} / \mathrm{min}$, and the injection volume was $10 \mu \mathrm{L}$. As shown in Figure 1, retention times of isoflavonoids were: $19.4 \mathrm{~min}$ in 6-hydroxygenistein 6, 7-di-O-glucoside, $20.2 \mathrm{~min}$ in glycitin, $21.4 \mathrm{~min}$ in tectorigenin 7-O-xylosylglucoside, $22.4 \mathrm{~min}$ in tectoridin, $26.5 \mathrm{~min}$ in glycitein, and $29.6 \mathrm{~min}$ in tectorigenin. Retention times of triterpenoid saponins were $35.4 \mathrm{~min}$ in soyasaponin I and $37.0 \mathrm{~min}$ in kaikasaponin III. According to the results of quantitative analysis by using a calibration curve of standard samples, thomsonide (100 mg) contained 3.99 mg 6-hydroxygenistein 6, 7-di-O-glucoside, $6.54 \mathrm{mg}$ glycitin, $11.84 \mathrm{mg}$ tectorigenin 7-O-xylosylglucoside, 5.82 


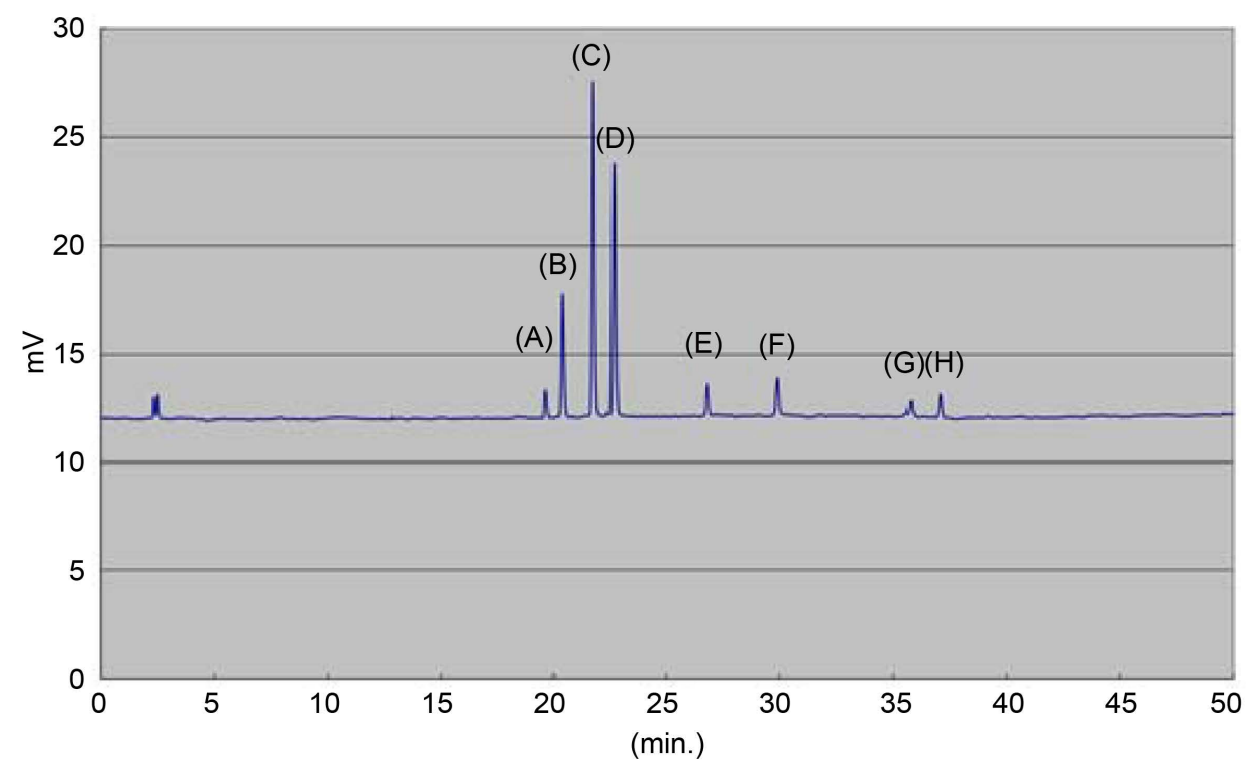

Figure 1. HPLC Chromatogram of Thomsonide. (A) 6-hydroxygenistein 6, 7-di-O-glucoside; (B) Glycitin; (C) Tectorigenin 7-O-xylosylglucoside; (D) Tectoridin; (E) Glycitein; (F) Tectorigenin; (G) Soyasaponin I; (H) Kaikasaponin III.

mg tectoridin, $2.39 \mathrm{mg}$ glycitein, and $2.34 \mathrm{mg}$ tectorigenin as isoflavonoids; $3.71 \mathrm{mg}$ soyasaponin I and $3.21 \mathrm{mg}$ kaikasaponin III as triterpenoid saponins.

\subsection{Animals}

Male Sprague-Dawley and Wistar strain rats weighing 180 - 200 g were obtained from Japan SLC, Inc. (Shizuoka, Japan). All animals were placed in cages, which were kept in an air-conditioned room with illumination from 07:00 to $19: 00 \mathrm{~h}$. Room temperature $\left(22^{\circ} \mathrm{C} \pm 2^{\circ} \mathrm{C}\right)$ and humidity $(55 \% \pm 10 \%)$ were controlled automatically. The animals were given free access to laboratory chow (pellets) (Funabashi Farm Co., Ltd, Chiba, Japan) and water. All procedures were in accordance with the Guiding Principles for the Care and Use of Laboratory Animals as adopted by the Japanese Pharmacological Society. The study protocol was approved by the Animal Ethics Committee of Ohta’s Isan Co., Ltd., Tokyo, Japan (20 January 2009; protocol No. 09-002).

\subsection{Drug Preparations}

Test compounds for oral administration were suspended in a vehicle containing Tween $80 \%$ and $1 \%$ sodium carboxymethylcellulose (CMC-Na) (1:19). The test compounds were administered by a feeding needle in a volume of $0.5 \mathrm{~mL}$ per $100 \mathrm{~g}$ body weight in the following concentrations: Thomsonide $100-400 \mathrm{mg} / \mathrm{kg}$ and geranylgeranylacetone (teprenone) (Wako Pure Chemical Industries, Ltd., Osaka, Japan) $20 \mathrm{mg} / \mathrm{kg}$. The concentration of each compound was chosen based on published literatures by Yamazaki et al. [5] and Watanabe et al. [8]. Thus, thomsonide was used at doses below $500 \mathrm{mg} / \mathrm{kg}$, because even at $5 \mathrm{~g} / \mathrm{kg}$, as a maximal practicable dose, it did not induce any marked change in behavior, except for the transient locomotor depression, nor lead to any deaths within $72 \mathrm{~h}$.

\subsection{Ethanol-Induced Gastric Mucosal Lesions in Intact Rats}

Fifty male Sprague-Dawley strain rats were fasted for $24 \mathrm{~h}$ prior to the experiment, but allowed free access to water. In accordance with the method of Robert et al. [9], thomsonide was orally administered to rats at doses of 100,200 , and $400 \mathrm{mg} / \mathrm{kg}$. Teprenone was orally administered to rats at a dose of $20 \mathrm{mg} / \mathrm{kg}$ as a positive control. The control group was treated with vehicles containing Tween 80\% and 1\% CMC-Na (1:19), instead of thomsonide. One hour later $1 \mathrm{~mL}$ of $99.5 \%$ ethanol was orally administered to the rats. One hour later, the rats were sacrificed under deep ether anesthesia, and the stomach was removed. After injecting $2 \%$ formalin into the sto- 
mach through the glandular portion, the stomach was fixed in $2 \%$ formalin for 10 minutes. It was then cut along the greater curvature rinsed with tap water, and was spread out on a sheet of white paper for examination. The length $(\mathrm{mm})$ of erosive lesions on the gastric mucosa was measured under a dissecting microscope, and the size of the gastric mucosal lesions was recorded as the sum of the lengths of the lesions.

\subsection{Prostaglandin (PG) Content of the Gastric Mucosa}

In the light of the method of Harada et al. [10], 24 male Wistar strain rats were fasted for $24 \mathrm{~h}$ prior to the experiment, but allowed free access to water. Thomsonide was orally administered to rats at doses of 200 and 400 $\mathrm{mg} / \mathrm{kg}$. Four hours later, the rats were sacrificed under deep ether anesthesia, and the stomach was removed immediately. The stomach was cut along the greater curvature and rinsed with ice-cold saline. In the light of the method of Powell [11], the corpus region was excised, weighed, and homogenized in $6 \mathrm{~mL}$ of $0.1 \mathrm{M}$ phosphate-buffered saline containing $10^{-5} \mathrm{M}$ indomethacin to prevent further formation of PGs, and then the homogenate was adjusted to $\mathrm{pH} 3$ with $1.5 \mathrm{~mL}$ of $2 \mathrm{M}$ hydrochloric acid. The homogenate was then centrifuged at $12,000 \times \mathrm{g}$ for $20 \mathrm{~min}$ at $4^{\circ} \mathrm{C}$, and the obtained supernatant was purified and was concentrated on Amprep $\mathrm{C}_{2}$ (100 mg resin, Amersham Japan, Tokyo Japan) to extract in methyl formate, according to the Amersham manual. After drying the specimens obtained in a centrifugal evaporator, they were used to assay PGs, and their prostaglandin $\mathrm{E}_{2}\left(\mathrm{PGE}_{2}\right)$ and 6-keto prostaglandin $\mathrm{F}_{1} \alpha\left(6-\mathrm{ketoPGF}_{1} \alpha\right)$ contents were determined by ELISA with a commercial kit (Cayman Chemical Company, USA).

\subsection{Ethanol-Induced Gastric Mucosal Lesions in Indomethacin-Pretreated Rats}

Forty male Sprague-Dawley strain rats were fasted for $24 \mathrm{~h}$ prior to the experiment, but allowed free access to water. In order to clarify whether the gastro protective effects of thomsonide may be related to the local enzymatic conversion of arachidonic acid to PGs, indomethacin (Sigma-Aldrich, Inc., St. Louis, MO, USA) suspended in vehicle (5 mL/kg) containing Tween 80 and saline (1:19) was injected subcutaneously into the rats at a dose of $10 \mathrm{mg} / \mathrm{kg}$, and thomsonide or teprenone was administered $30 \mathrm{~min}$ later. A volume of $1 \mathrm{~mL}$ at $99.5 \%$ ethanol was orally administered to the rats $1 \mathrm{~h}$ after administration of thomsonide or teprenone. One hour later, the rats were sacrificed under deep ether anesthesia, and the stomach was removed. The rest of the procedure was as described above.

\subsection{Statistical Analysis}

The data are expressed as means \pm standard error of the mean (S.E.M). Data were evaluated for significant differences by the one-way analysis of variance (ANOVA) and Dunnet's multiple range test.

\section{Results}

\subsection{Ethanol-Induced Gastric Mucosal Lesions in Intact Rats}

One hour after administration of $1 \mathrm{~mL}$ of 99.5\% ethanol, erosive mucosal lesions with congestion were observed in the stomach (Figure 2(a)). The lesions were markedly reduced by the pretreatment with thomsonide or teprenone (Figure 2(b) and Figure 2(c)). As shown in Table 1, the length of the lesions was $109.10 \pm 8.29 \mathrm{~mm}$ in the vehicle-treated rats, i.e., the control group. Pretreatment with thomsonide $60 \mathrm{~min}$ before ethanol administration inhibited dose-dependently the gastric lesions at doses ranging from 100 to $400 \mathrm{mg} / \mathrm{kg}$. At doses of 200 $\mathrm{mg} / \mathrm{kg}$ and above the effect was marked compared to the control group. In addition, pretreatment with teprenone at a dose of $20 \mathrm{mg} / \mathrm{kg}$ inhibited more significantly the gastric lesions compared to the control group.

Accordingly, thomsonide, as well as teprenone, had protective effects against ethanol-induced gastric lesions.

\subsection{Prostaglandin (PG) Content of the Gastric Mucosa}

As shown in Table 2, the levels of $\mathrm{PGE}_{2}$ and 6-ketoPGF $\alpha$ in gastric mucosa were $50.23 \pm 5.81 \mathrm{ng} / \mathrm{g}$ and $385.38 \pm$ $112.08 \mathrm{ng} / \mathrm{g}$, respectively, in the vehicle-treated group. Thomsonide increased the $\mathrm{PGE}_{2}$ and 6-ketoPGF $\alpha$ contents of the gastric mucosa, and the increases at doses of $200 \mathrm{mg} / \mathrm{kg}$ and above were significantly compared to those in the vehicle-treated group. Thomsonide significantly increased the $\mathrm{PGE}_{2}$ and 6-ketoPGF $\alpha$ contents of the gastric mucosa. 


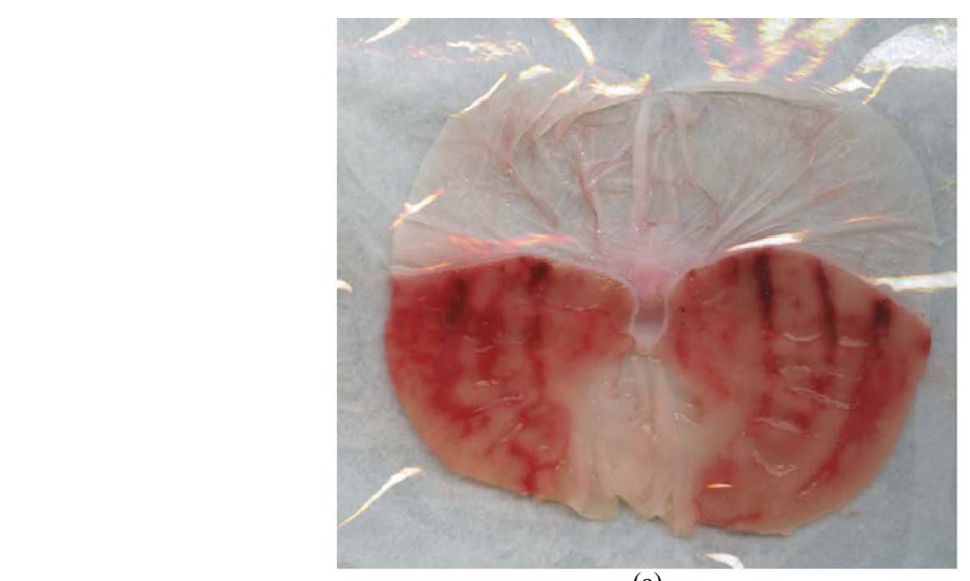

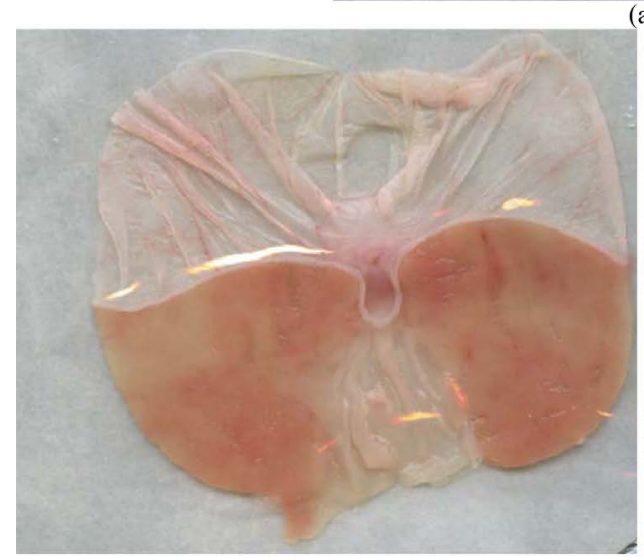

(b) (a)

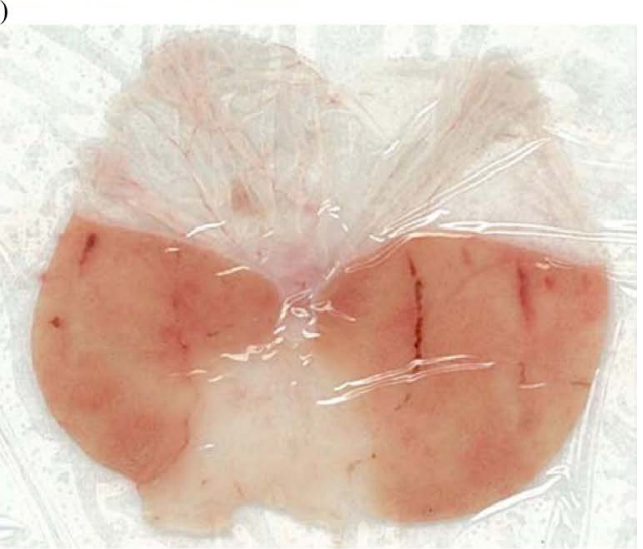

(c)

Figure 2. (a) Gross appearance of gastric mucosa $1 \mathrm{~h}$ after ethanol administration (Vehicle + ethanol). Erosive congestion was observed in the fundus. (b) Gross appearance of gastric mucosa $1 \mathrm{~h}$ after ethanol administration Pretreatment with Thomsonide at a dose of $400 \mathrm{mg} / \mathrm{kg}$ reduced the erosive congestion. (c) Gross appearance of gastric mucosa $1 \mathrm{~h}$ after ethanol administration Pretreatment with Tepreone at a dose of $20 \mathrm{mg} / \mathrm{kg}$ reduced the erosive congestion.

Table 1. Effects of thomsonide and teprenone on ethanol-induced gastric lesions in intact rats.

\begin{tabular}{ccccc}
\hline & & N & Gastric lesions (mm) \\
\hline Vehicle & & 10 & $109.10 \pm 8.29$ \\
& $100 \mathrm{mg} / \mathrm{kg}$ & 10 & $90.60 \pm 14.73$ & 16.96 \\
Thomsonide & $200 \mathrm{mg} / \mathrm{kg}$ & 10 & $64.70 \pm 7.93^{* *}$ & $24.90 \pm 6.91^{* *}$ \\
& $400 \mathrm{mg} / \mathrm{kg}$ & 10 & $42.80 \pm 10.57^{* *}$ & 77.18 \\
Teprenone & $20 \mathrm{mg} / \mathrm{kg}$ & 10 & 60.77 & \\
\hline
\end{tabular}

${ }^{* *} p<0.01$ (ANOVA and Dunnet’s multiple range test) significantly different from control.

Table 2. Effects of thomsonide on $\mathrm{PGE}_{2}$ and 6-ketoPGF $\mathrm{P}_{1} \alpha$ content of gastric mucosa in rats.

\begin{tabular}{ccccc}
\hline & & $\mathrm{N}$ & $\mathrm{PGE}_{2}(\mathrm{ng} / \mathrm{g})$ & 6-ketoPGF $\alpha(\mathrm{ng} / \mathrm{g})$ \\
\hline Vehicle & & 8 & $50.23 \pm 5.81$ & $385.38 \pm 112.08$ \\
Thomsonide & $200 \mathrm{mg} / \mathrm{kg}$ & 8 & $90.92 \pm 9.25^{* *}$ & $847.16 \pm 74.41^{* *}$ \\
& $400 \mathrm{mg} / \mathrm{kg}$ & 8 & $90.82 \pm 6.30^{* *}$ & $980.08 \pm 69.39^{* *}$ \\
\hline
\end{tabular}

${ }^{* *} p<0.01$ (ANOVA and Dunnet’s multiple range test) significantly different from Vehicle treated group.

\subsection{Ethanol-Induced Gastric Mucosal Lesions in Indomethacin-Pretreated Rats}

Gastric mucosal lesions were also observed in rats, which were treated with ethanol following indomethacin pre- 
treatment (Figure 3(a)). As shown in Table 3, in rats treated with indomethacin, the ethanol-induced gastric lesions measured $115.90 \pm 14.05 \mathrm{~mm}$ in the vehicle-treated group, as opposed to $107.30 \pm 17.41$ and $78.00 \pm 13.70$ $\mathrm{mm}$ in the thomsonide groups given the $200 \mathrm{mg} / \mathrm{kg}$ dose and $400 \mathrm{mg} / \mathrm{kg}$ dose, respectively. The ethanol-induced gastric lesions in the indomethacin-pretreated rats measured $79.60 \pm 9.45 \mathrm{~mm}$ in the teprenone group given the $20 \mathrm{mg} / \mathrm{kg}$ dose. The differences in gastric lesions between the thomsonide group or teprenone group and the vehicle-treated group were not significant, although these lesions were barely reduced by thomsonide or teprenone pretreatment (Figure 3(b) and Figure 3(c)). Pretreatment with indomethacin attenuated the protective effect of thomsonide, as well as teprenone, against the ethanol-induced gastric lesions.

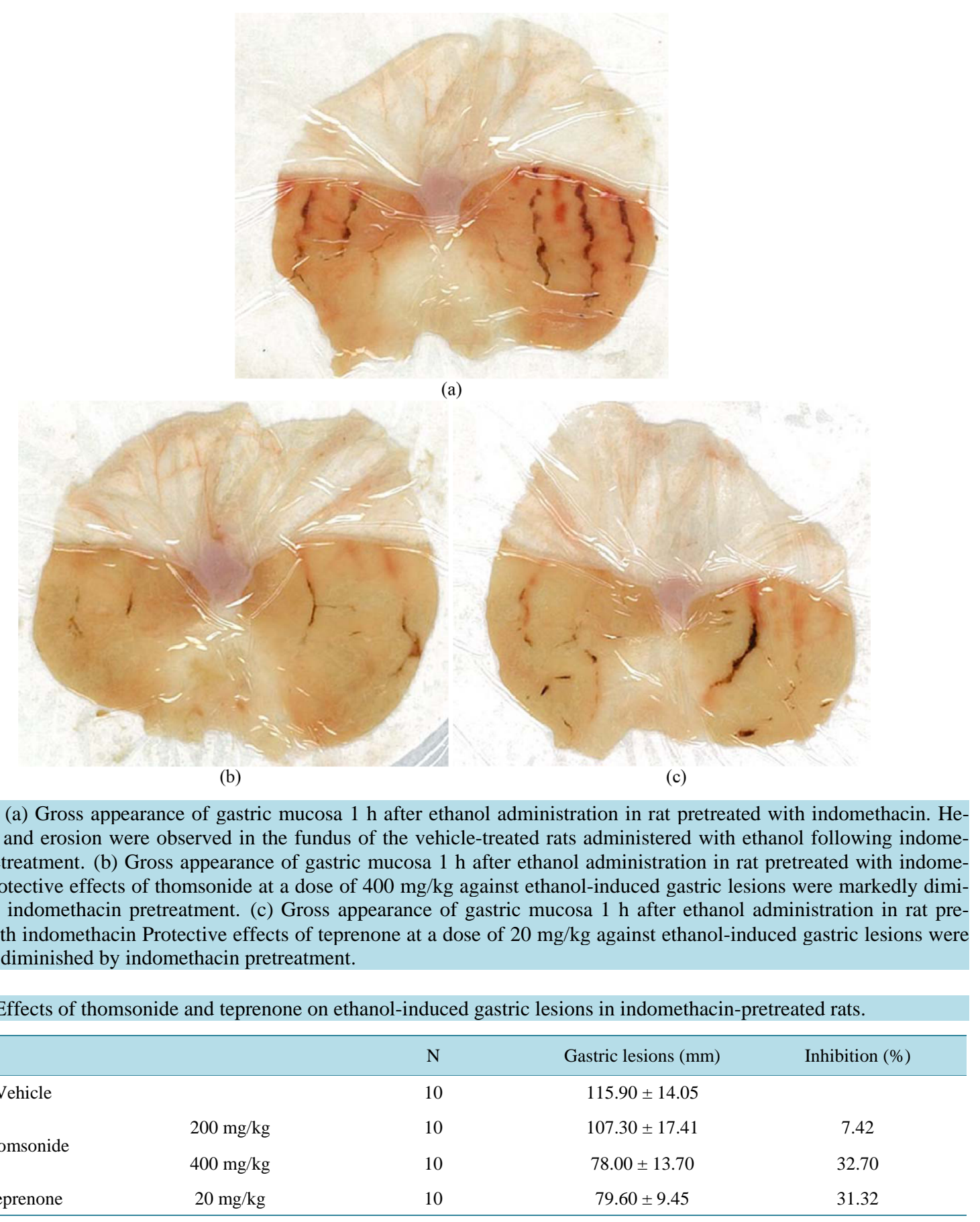

Figure 3. (a) Gross appearance of gastric mucosa $1 \mathrm{~h}$ after ethanol administration in rat pretreated with indomethacin. Hemorrhage and erosion were observed in the fundus of the vehicle-treated rats administered with ethanol following indomethacin pretreatment. (b) Gross appearance of gastric mucosa $1 \mathrm{~h}$ after ethanol administration in rat pretreated with indomethacin. Protective effects of thomsonide at a dose of $400 \mathrm{mg} / \mathrm{kg}$ against ethanol-induced gastric lesions were markedly diminished by indomethacin pretreatment. (c) Gross appearance of gastric mucosa $1 \mathrm{~h}$ after ethanol administration in rat pretreated with indomethacin Protective effects of teprenone at a dose of $20 \mathrm{mg} / \mathrm{kg}$ against ethanol-induced gastric lesions were markedly diminished by indomethacin pretreatment.

Table 3. Effects of thomsonide and teprenone on ethanol-induced gastric lesions in indomethacin-pretreated rats.

\begin{tabular}{ccccc}
\hline & & N & Gastric lesions (mm) & Inhibition (\%) \\
\hline Vehicle & & 10 & $115.90 \pm 14.05$ & \\
Thomsonide & $200 \mathrm{mg} / \mathrm{kg}$ & 10 & $107.30 \pm 17.41$ & 7.42 \\
& $400 \mathrm{mg} / \mathrm{kg}$ & 10 & $78.00 \pm 13.70$ & 32.70 \\
Teprenone & $20 \mathrm{mg} / \mathrm{kg}$ & 10 & $79.60 \pm 9.45$ & 31.32 \\
\hline
\end{tabular}

Not significantly different from Vehicle. 


\section{Discussion}

No reports have discussed the gastro protective effect of Puerariae flos, although preparations of this plant have been used in the traditional Chinese herbal medicine to counteract the effects of alcohol consumption. In the present study we found that thomsonide, an extract of Puerariae flos, as well as teprenone, had a protective effect against ethanol-induced gastric mucosal lesions. The results of our present study also showed that thomsonide significantly increased $\mathrm{PGE}_{2}$ and 6-ketoPGF ${ }_{1} \alpha$ contents of the gastric mucosa, and that pretreatment with indomethacin attenuated the protective effect of thomsonide against ethanol-induced gastric mucosal lesions. Furthermore, our present results showed that the protective effect of teprenone against ethanol-induced gastric mucosal lesions, was attenuated by pretreatment with indomethacin. Some reports have already shown that teprenone has a protective effect against ethanol-induced gastric mcosal lesions [8] and increases the $\mathrm{PGE}_{2}$ content of the gastric mucosa through induction of COX-2 [12]. These findings are generally consistent with our observations. A variety of PGs have been shown to protect the gastrointestinal mucosa against various noxious agents. Robert et al. [9] have described the protective property of PGs, which is independent of their acid-inhibiting property, as "adaptive cytoprotection". $\mathrm{PGE}_{2}$ and $\mathrm{PGI}_{2}$ are well known to be involved in the regulation of a variety of gastrointestinal functions. Araki et al. [13] have reported that endogenous $\mathrm{PGE}_{2}$ protects the gastric mucosa through the $\mathrm{EP}_{1}$ receptor. The above findings suggest that the gastro protective effects of thomsonide may be related to the cytoprotective activity of endogenous $\mathrm{PGE}_{2}$ through the $\mathrm{EP}_{1}$ receptor. In addition, thomsonide increased the gastric mucosal content of 6-ketoPGF $1 \alpha$, a stable metabolite of $\mathrm{PGI}_{2}$, indicating that thomsonide has a significant effect on the production of $\mathrm{PGI}_{2}$. Konturek et al. [14] have reported that $\mathrm{PGI}_{2}$ increases the postprandial serum gastrin level and mucosal blood flow in the resting mucosa in dogs with Heidenhain pouches; Gaskill et al. [15] have reported that $\mathrm{PGI}_{2}$ increases gastric mucosal blood flow by the mechanism, which is dependent on cyclic AMP. In light of these findings, thomsonide may protect ethanol-induced gastric mucosal lesions by maintaining gastric blood flow through the increase in the gastric mucosal content of $\mathrm{PGI}_{2}$, a precursor of 6-ketoPGF $1 \alpha$, as well as the cytoprotective activity of endogenous $\mathrm{PGE}_{2}$.

Puerariae flos is known to be rich in isoflavonoids and triterpenoid saponins. Kinjo et al. [16] have reported that Puerariae flos contains a variety of triterpenoid saponins including Kakkasaponin I. Matsuda et al. [17] have reported that oleanene-type triterpenoid saponins have a protective effect against ethanol-induced gastric mucosal lesions. Martinez et al. [18] have also reported that oleanolic acid can be regarded as a bioactive molecule, which may induce PGs release in the COX-2-dependent manner. In view of the above evidence, it is plausible that thomsonide, which is rich in oleanene-type triterpenoid saponins, has a protective effect against the formation of gastric mucosal lesions. It is supposed that Kakkasaponin, a component of thomsonide, may protect the gastric mucosa from ethanol-induced lesions by increasing the production of endogenous PGs through overexpression of COX-2 in the gastric mucosa. The results of the present study prove our supposition that thomsonide has a gastro protective effect, and that the protective effect of thomsonide may be due to the local enzymatic conversion of arachidonic acid to PGs, which are known to have cytoprotective properties. The results also suggest that the gastro protective effect of thomsonide may partially mitigate alcoholic disorders in the gastrointestinal tract. Furthermore, the results support our pharmacological belief that Puerariae flos is useful for the treatment of alcoholic disorders.

\section{Conclusion}

Thomsonide, an extract of Puerariae flos, has been investigated for its effect on ethanol-induced gastric mucosal lesions. Thomsonide, as well as teprenone, inhibited dose-dependently the formation of ethanol-induced gastric mucosal lesions. At doses of $200 \mathrm{mg} / \mathrm{kg}$ and above, thomsonide produced the significant effect, and increased significantly the $\mathrm{PGE}_{2}$ and 6-ketoPGF $1 \alpha$ contents of the gastric mucosa. Furthermore, indomethacin attenuated the protective effect of thomsonide, as well as teprenone, against the formation of ethanol-induced gastric mucosal lesions. These results elucidate that thomsonide has a gastro protective effect and that its protective effect may be produced by increasing endogenous PGs in gastric mucosa.

\section{References}

[1] (1985) Chinese Drug Dictionary. Shogakukan, Tokyo, 551-552.

[2] Kubo, M., Sasaki, M., Namba, K., Naruto, S. and Nishimura, H. (1975) Isolation of a New Isoflavone from Chinese 
Pueraria Flowers. Chemical Pharmaceutical Bulletin, 23, 2449-2451. http://dx.doi.org/10.1248/cpb.23.2449

[3] Kinjo, J., Aoki, K., Okawa, M., Shii, Y., Hirakawa, T., Nohara, T., Nakajima, Y., Yamazaki, T., Hosono, T., Niiho, Y. and Kurashige, T. (1999) HPLC Profile Analysis of Hepatoprotective Oleanene-Glucuronides in Puerariae flos. Chemical Pharmaceutical Bulletin, 47, 708-710. http://dx.doi.org/10.1248/cpb.47.708

[4] Yamazaki, T., Hosono, T., Matsushita, Y., Kawashima, K., Someya, M., Nakajima, Y., Narui, K., Hibi, Y., Ishizaki, M., Kinjo, J. and Nohara, T. (2002) Pharmacological Studies on Puerariae flos IV: Effects of Pueraria Thomsonii Dried Flower Extracts on Blood Ethanol and Acetaldehyde Levels in Humans. International Journal of Clinical pharmacology Research, XXII, 23-28.

[5] Yamazaki, T., Yaguchi, M., Nakajima, Y., Hosono, T., Niiho, Y., Hibi, Y., Kinjo, J. and Nohara, T. (2005) Effects of an Aqueous Extract of Puerariae flos (Thomsonide) on Impairment of Passive Avoidance Behavior in Mice. Journal of Ethnopharmacology, 100, 244-248. http://dx.doi.org/10.1016/j.jep.2005.02.034

[6] Terano, A., Shiga, J., Hiraishi, H., Ota, S. and sugimoto, T. (1986) Protective Action of Tetraprenylactone against Ethanol-Induced Damage in Rat Gastric Mucosa. Digestion, 35, 182-188. http://dx.doi.org/10.1159/000199365

[7] Niiho, Y., Nakajima, Y., Yamazaki, T., Okamoto, M., Tsuchihashi, R., Kodera, M., Kinjo, J. and Nohara, T. (2010) Simultaneous Analysis of Isoflavones and Saponins in Pueraria Flowers Using HPLC Coupled to an Evaporative Light Scattering Detector and Isolation of a New Isoflavone Diglucoside. Journal of Natural Medicines, 64, 313-320. http://dx.doi.org/10.1007/s11418-010-0411-z

[8] Watanabe, R., Katoh, Y., Tanaka, M. and Tajima, T. (1988) Effects of Selbex 10\% on Ethanol-Induced Gastric Damages in Rats. Basic and Clinical Report, 22, 6353-6358.

[9] Robert, A., Nezamis, J.E., Lancaster, C. and Hanchar, A.J. (1979) Cytoprotection by Prostaglandins in Rats. Prevention of Gastric Necrosis Produced by Alcohol, $\mathrm{HCl}, \mathrm{NaOH}, \mathrm{Hypertonic} \mathrm{NaCl,} \mathrm{and} \mathrm{Thermal} \mathrm{Injury.} \mathrm{Gastroenterology,} \mathrm{77,}$ 433-443.

[10] Harada, N., Okajima, K., Murakami, K., Isobe, H. and Liu, W. (1999) Gastric Prostacyclin (PGI $)$ Prevents StressInduced Gastric Mucosal Injury in Rats Primarily by Inhibiting Leukocyte Activation. Prostaglandins \& other Lipid Mediators, 57, 291-303. http://dx.doi.org/10.1016/S0090-6980(98)00077-X

[11] Powell, W.S. (1980) Rapid Extraction of Oxygenated Metabolites of Arachidonic Acid from Biological Samples Using Octadecylsilyl Silica. Prostaglandins, 20, 947-957. http://dx.doi.org/10.1016/0090-6980(80)90144-6

[12] Yabe, Y., Fu, Y., Kawano, J. and Tsuji, S. (2004) Geranylgeranylacetone Enhances COX-2 Expression and PGE2 Production via NF-кB in RGM1 Cells. Ulcer Research, 31, 151-153.

[13] Araki, H., Ukawa, H., Sugawa, Y., Yagi, K., Suzuki, K. and Takeuchi, K. (2000) The Roles of Prostaglandin E Receptor Subtypes in the Cytoprotective Action of Prostaglandin $\mathrm{E}_{2}$ in Rat Stomach. Alimentary Pharmacology and Therapeutics, 14, 116-124. http://dx.doi.org/10.1046/j.1365-2036.2000.014s1116.x

[14] Konturek, S.J., Robert, A., Hanchar, A.J. and Nezamis, J.E. (1980) Comparison of Prostacyclin and Prostaglandin E ${ }_{2}$ on Gastric Secretion, Gastrin Release, and Mucosal Blood Flow in Dogs. Digestive Diseases and Sciences, 25, 673-679. http://dx.doi.org/10.1007/BF01308326

[15] Gaskill, H.V., Sirinek, K.R. and Levine, B.A. (1982) Prostacyclin Increases Gastric Mucosal Blood Flow via Cyclic AMP. Journal of Surgical Research, 33, 140-145. http://dx.doi.org/10.1016/0022-4804(82)90020-8

[16] Kinjo, J., Takeshita, T., Abe, Y., Terada, N., Yamashita, H., Yamasaki, M., Takeuchi, K., Murakami, K., Tomimatsu, T. and Nohara, T. (1988) Studies on the Constituents of Pueraria lobata. IV. Chemical Constituents in the Flowers and the Leaves. Chemical Pharmaceutical Bulletin, 36, 1174-1179. http://dx.doi.org/10.1248/cpb.36.1174

[17] Matsuda, H., Li, Y., Murakami, T., Yamahara, J. and Yoshikawa, M. (1998) Protective Effects of Oleanolic Acid Oligoglycosides on Ethanol- or Indomethacin-Induced Gastric Mucosal Lesions in Rats. Life Sciences, 63, 245-250. http://dx.doi.org/10.1016/S0024-3205(98)00426-3

[18] Martinez-Gonzalez, J., Rodriguez-Rodriguez, R., Gonzalez-Diez, M., Rodriguez, C., Herrera, M.D., Ruiz-Gutierrez, V. and Badimon, L. (2008) Oleanolic Acid Induces Prostacyclin Release in Human Vascular Smooth Muscle Cells through a Cyclooxygenase-2-Dependent Mechanism. Journal of Nutrition, 138, 443-448. 